\title{
Chirality flip of Weyl nodes and its manifestation in strained $\mathrm{MoTe}_{2}$
}

\author{
Viktor Könye $\odot,{ }^{1}$ Adrien Bouhon $\odot,{ }^{2}$ Ion Cosma Fulga $\odot,{ }^{1}$ Robert-Jan Slager $\odot,{ }^{3}$ Jeroen van den Brink $\odot,{ }^{1,4}$ \\ and Jorge I. Facio $\circledast^{1}$ \\ ${ }^{1}$ Institute for Theoretical Solid State Physics, IFW Dresden and Würzburg-Dresden Cluster of Excellence ct.qmat, \\ Helmholtzstr. 20, 01069 Dresden, Germany \\ ${ }^{2}$ Nordic Institute for Theoretical Physics (NORDITA), Hannes Alfvéns väg 12, 10691 Stockholm, Sweden \\ ${ }^{3}$ TCM Group, Cavendish Laboratory, University of Cambridge, J. J. Thomson Avenue, Cambridge CB3 OHE, United Kingdom \\ ${ }^{4}$ Institute for Theoretical Physics, TU Dresden, 01069 Dresden, Germany
}

(Received 9 July 2021; accepted 15 October 2021; published 5 November 2021)

\begin{abstract}
Due to their topological charge, or chirality, the Weyl cones present in topological semimetals are considered robust against arbitrary perturbations. One well-understood exception to this robustness is the pairwise creation or annihilation of Weyl cones, which involves the overlap in energy and momentum of two oppositely charged nodes. Here we show that the topological charge can in fact change sign, in a process that involves the merging of not two, but three Weyl nodes. This is facilitated by the presence of rotation and time-reversal symmetries, which constrain the relative positions of Weyl cones in momentum space. We analyze the chirality flip process, showing that transport properties distinguish it from the conventional, double Weyl merging. Moreover, we predict that the chirality flip occurs in $\mathrm{MoTe}_{2}$, where experimentally accessible strain leads to the merging of three Weyl cones close to the Fermi level. Our work sets the stage to further investigate and observe such chirality flipping processes in different topological materials.
\end{abstract}

DOI: 10.1103/PhysRevResearch.3.L042017

Introduction. Weyl semimetals are arguably the most robust form of gapless topological matter [1-9]. They host pointlike degeneracies of their energy bands, called Weyl nodes (or cones), whose low energy, linear dispersion relation is similar to that of elementary particles called Weyl fermions [10]. These band touching points are not accidental, but are a manifestation of the topologically nontrivial character of the semimetal phase. Each node has a chirality associated to it, a topological charge given by its Chern number, which means that an isolated Weyl cone cannot be gapped out by any perturbation. Instead, it can only be moved in energy and momentum space, or tilted [11]. This property sets Weyl semimetals apart from the many other types of gapless topological matter, in which degenerate points [12-17] (or lines $[5,18]$ ) require additional symmetries in order to remain protected.

Provided that Weyl cones are not isolated, it is possible to change their number: they can be created or annihilated pairwise when they overlap in energy and momentum. Similar to electrodynamics, these two-node processes require "topological charge conservation," meaning that the total Chern number of all band touching points in the Brillouin zone (BZ) must vanish [1]. The pairwise creation and annihilation of Weyl cones has been studied extensively, especially as a method to engineer Weyl semimetal phases by applying per-

Published by the American Physical Society under the terms of the Creative Commons Attribution 4.0 International license. Further distribution of this work must maintain attribution to the author(s) and the published article's title, journal citation, and DOI. turbations such as strain [19-22], magnetic field or changes in magnetization direction [23-26], disorder [27-30], phonons [31], or high-frequency illumination [32,33] to real materials.

In theory, there is no constraint limiting the number of Weyl nodes that can merge at a given point in the BZ. In practice, however, processes involving the simultaneous overlap of three or more cones of different chirality are highly improbable, especially when each node is allowed to occupy any point in energy and momentum space. To date, multi-Weyl merging has been studied mainly in the context of rather exotic types of topological semimetals [14,34], hosting "unconventional fermions." There, the symmetry-protected band degeneracies may split into multiple Weyl nodes if very particular symmetries are broken $[14,35,36]$.

Here we study simple three-node processes occurring purely inside a Weyl semimetal phase, without unconventional fermions: a single Weyl cone overlaps with a pair of oppositely charged ones, causing the latter to disappear from the band structure. By topological charge conservation, the result is a single node with a flipped chirality. Interestingly, while lattice symmetries are not responsible for the existence of the degeneracy points, they play a fundamental role in enabling the three-node process to occur. This is because they restrict the relative positions and charges of the nodes in the BZ (for instance, to planes or lines). Throughout the following, we will focus on systems obeying a combination of twofold rotation and time-reversal symmetry.

One of the advantages of studying multinode processes in Weyl semimetals as opposed to more exotic types of gapless topological matter is that Weyl semimetal phases are much more abundant in real materials [37]. In fact, we find that one of the earliest predicted Weyl materials, $\mathrm{MoTe}_{2}$ [38], hosts 
very close to the Fermi level a three-node process upon applying small uniaxial strain. As a result of this process, a Weyl node in a high-symmetry plane flips its chirality. This shows that the three-node process is within reach of photoemission experiments. Further, it shows that strain in real materials can act as a "chirality switch," allowing one to tune the transport properties associated with gapless topological systems.

Chirality flip process in simple models. We start by discussing how the chirality of a Weyl node can flip using toy models. Given the requirement of topological charge conservation and considering only simple Weyl cones, chirality flips are only possible if at least two other, oppositely charged Weyl nodes are involved. For concreteness, we consider processes that start from three Weyl nodes with Chern numbers $\mathcal{C}=+1$, $-1,-1$, and end with a single Weyl node having $\mathcal{C}=-1$ [39]. We find that there are two scenarios through which this can happen: one that involves a three-node process and one that only involves two-node processes.

We begin by discussing the first scenario, corresponding to a three-node process, meaning that all three Weyl cones simultaneously overlap at the same point in the BZ. While this merging could in principle occur at generic momenta, its likelihood can be greatly increased when symmetries constrain the relative positions and charges of the band touching points. As mentioned above, we will consider a continuum model invariant under the combination of twofold rotation and time-reversal symmetries, with Hamiltonian

$$
H_{1}(\mathbf{k})=k_{x} \sigma_{x}+\left(\alpha k_{z}+k_{z}^{3}\right) \sigma_{y}+k_{y} \sigma_{z} .
$$

Here, $\mathbf{k}=\left(k_{x}, k_{y}, k_{z}\right)$ is the quasimomentum and $\sigma_{i}$ are Pauli matrices encoding the degree of freedom associated with the two bands. For simplicity, we will set units such that the Hamiltonian and the quasimomentum are dimensionless.

The Hamiltonian Eq. (1) obeys a twofold rotation symmetry along the $k_{x}=k_{y}=0$ axis, with operator $C_{2}=-i \sigma_{y}$, as well as time-reversal symmetry $T=i \sigma_{y} K$ (where $K$ is complex conjugation), such that

$$
\begin{aligned}
T H_{1}(\mathbf{k}) T^{\dagger} & =H_{1}(-\mathbf{k}), \\
C_{2} H_{1}(\mathbf{k}) C_{2}^{\dagger} & =H_{1}\left(-k_{x},-k_{y}, k_{z}\right) .
\end{aligned}
$$

Their combination $C_{2} T=K$ implies that

$$
H_{1}^{*}\left(k_{x}, k_{y}, k_{z}\right)=H_{1}\left(k_{x}, k_{y},-k_{z}\right),
$$

and that the Hamiltonian is real for $k_{z}=0$.

The constraint Eq. (4) means that, if a Weyl cone is present in the $C_{2} T$ invariant plane, $k_{z}=0$, then it cannot exit the plane, due to topological charge conservation. Further, if there is a Weyl node at $k_{z}>0$, another one must be positioned symmetrically at $k_{z}<0$. Note that pairs of out-of-plane cones must have the same Chern number, since both time-reversal and twofold rotation are charge preserving.

For $\alpha<0, H_{1}$ hosts three Weyl cones, a positive chirality node at the $\Gamma$ point, $\mathbf{k}=0$, and two negative chirality nodes at $\mathbf{k}=(0,0, \pm \sqrt{|\alpha|})$. In contrast, for $\alpha>0$ there is a single Weyl node with negative chirality at $\Gamma$. As shown in Figs. 1(a) and 1(b), changing the parameter $\alpha$ continuously from negative to positive values causes the three nodes to merge at the same point, such that the central node flips its chirality.
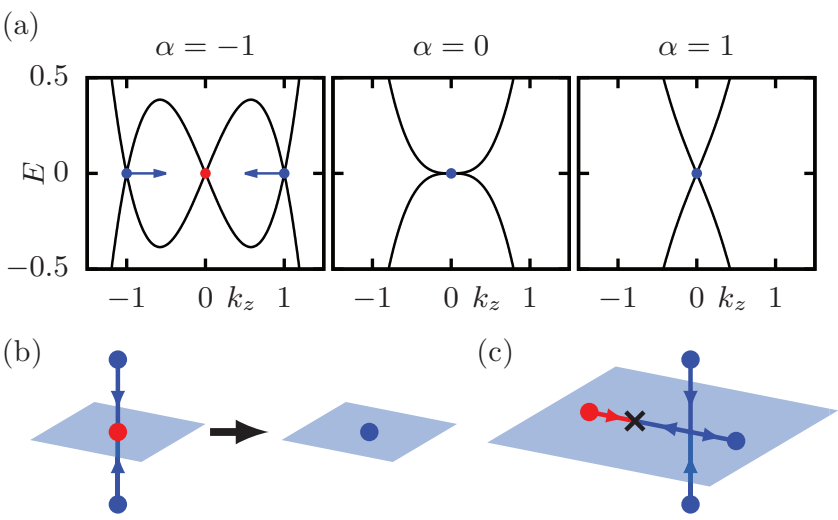

FIG. 1. Schematic representation of the Weyl node processes. The red (blue) points represent positive (negative) chirality Weyl nodes, the arrows represent their trajectories upon increasing $\alpha$, and the $C_{2} T$-invariant plane is marked in blue. (a) Band structure of Eq. (1) plotted at $k_{x}=k_{y}=0$ for three values of $\alpha$. (b) Chirality flip with a three-node process, as occurring in $H_{1}$. (c) Chirality flip with two-node processes, as happens in the Hamiltonian $\mathrm{H}_{2}$.

The three-node process occurring in the band structure of $H_{1}$ is protected by symmetry: the Weyl merging must involve all three nodes simultaneously. This is because the Hamiltonian obeys $C_{2}$ and $T$ separately, and because all Weyl nodes are located on the twofold rotation axis. The band touching points cannot move away from $k_{x, y}=0$ without breaking the rotation symmetry, and they must be positioned symmetrically with respect to $k_{z}$ due to time-reversal symmetry. The only allowed overlap is thus at $\Gamma$.

The presence of a chirality flip in $H_{1}$ is also related to topological quantities. Due to the presence of $C_{2} T$, the Hamiltonian is real at $k_{z}=0$. Therefore, a closed momentumspace loop in this plane will be characterized by a $\pi$-quantized Berry phase, provided that the loop encircles the central node. This $\mathbb{Z}_{2}$ topological invariant indicates that the parity of nodes inside the loop must be conserved [40,41]. Therefore, the central node is not allowed to leave the plane, but it is allowed to reverse its chirality.

If the system instead hosts Weyl cones away from the rotation axis, or if it obeys only the combined $C_{2} T$ symmetry but not its individual components, the three-node process is not symmetry protected anymore. This does not mean that it is forbidden. Even if it is not forced to occur, it still can, and as we show later actually does happen in real materials.

We now move on to discuss the second scenario for the chirality flip, one that does not involve a three-node process. We illustrate this scenario in Fig. 1(c), which shows the Weyl points of the Hamiltonian

$$
H_{2}(\mathbf{k})=k_{y} \sigma_{x}+k_{x} k_{z} \sigma_{y}+\left(k_{x}^{2}+k_{x}^{3}-k_{z}^{2}-\alpha\right) \sigma_{z} .
$$

Note that $H_{2}$ obeys the same constraint as $H_{1}$ in Eq. (4), but has neither rotation nor time-reversal symmetries.

For $\alpha<0$ there are three Weyl nodes in the band structure of $H_{2}$ : one in the $C_{2} T$ invariant plane $\mathbf{k}_{\mathbf{c}}=\left(k_{0}, 0,0\right)$, where $k_{0}$ is the single real solution of $k^{3}+k^{2}=-|\alpha|$, and two away from the $C_{2} T$ plane at $\mathbf{k}_{\mathrm{s}}=(0,0, \pm \sqrt{|\alpha|})$. Due to $C_{2} T$, the outside nodes can only enter the plane at the same point, as before. However, now they can meet without the third node, 
thus forming a double Weyl point. In $\mathrm{H}_{2}$, the double Weyl point occurs at $k_{z}=0$ when $\alpha=0$. For $\alpha \gtrsim 0$ the overlapping nodes separate again, and all three cones are in the $C_{2} T$ plane, located at $\left(k_{i}, 0,0\right)$, where $k_{i}$ are the real solutions of $k^{3}+$ $k^{2}=|\alpha|$. The three nodes remain until $\alpha=4 / 27$, at which point two of the opposite chirality Weyl points annihilate. At $\alpha>4 / 27$, we are left with a single Weyl node. The trajectory of all three Weyl cones is shown schematically in Fig. 1(c). Similar to the behavior of nodes in the spectrum of $H_{1}$, going from negative to positive $\alpha$ converts a positive chirality Weyl point into a negative chirality one.

As before, considering a momentum-space loop in the $C_{2} T$ plane that encloses the projections of all nodes in Fig. 1(c), we find a $\pi$-quantized Berry phase for all $\alpha$. This indicates that the two-node and three-node process cannot be distinguished topologically by using the Berry phase. This is expected, as one can imagine continuously moving the meeting point of the out-of-plane nodes until this point overlaps with the $k_{z}=$ 0 node. However, when considering an infinitesimally small loop around the initial red node, a blue node will have to cross this loop for some $\alpha$, thus ensuring a discontinuity in the Berry phase.

We note that $C_{2} T$ symmetry also relates to the Euler class [42-48], which quantifies the obstruction for pairs of Weyl nodes to leave the $C_{2} T$ plane. For a generic, multiband system obeying $C_{2} T$ symmetry, this obstruction is expressed by an additional set of topological invariants, independent of the Chern numbers, which characterize Weyl nodes inside the $k_{z}=0$ plane. These additional invariants are called "non-Abelian frame charges," and we denote them by $q$. A pair of nodes with opposite non-Abelian charges have a Euler class of zero, and a pair of nodes with the same non-Abelian charge carry a nonzero Euler class. Importantly, if the Euler class is nonzero, then the two nodes are not allowed to exit the plane, neither by merging and moving away from $k_{z}=0$ nor by annihilating with each other.

In our two-band models, these additional invariants $q$ are given simply by the winding number of the vector multiplying the $\sigma$ matrices [45], and take values $q= \pm 1$. For the twonode process shown in Fig. 1(c), the Euler class has several consequences. First, the out-of-plane nodes (blue) must acquire opposite non-Abelian charges $(q=+1$ and $q=-1)$ once they enter the plane, reflecting the fact that this pair is allowed to leave the $C_{2} T$ plane again, through the reversed process. Further, the annihilation of the initial in-plane node (red) with one of two nodes that entered the plane tells us that they must have opposite non-Abelian charges. Therefore, the non-Abelian charge $q$ of the remaining node must be the same as that of the initial in-plane node at the beginning of the process. In other words, even though the Weyl cone flips its chirality, the non-Abelian charge associated to it remains conserved. There could be no annihilation if the nodes of equal chirality (blue) were swapped, since the two merging nodes would then have the same value of $q$.

Transport properties. The initial and final stages of both chirality flipping processes are the same. This means that the observables for large $|\alpha|$ have the same qualitative behavior for the two processes. The difference in the two scenarios is prominent close to $\alpha=0$, where the two Weyl nodes outside of the $C_{2} T$ invariant plane reach the plane.
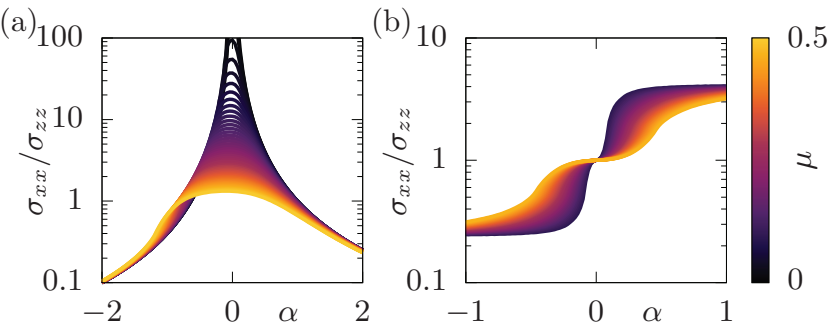

FIG. 2. Ratio of conductivities in the plane of the three Weyl nodes as a function of the parameter $\alpha$ for different chemical potentials $\mu$. Panel (a) shows the three-node process and (b) shows the two-node process.

We study the differences between the two processes by computing the conductivity using semiclassical Boltzmann transport theory in the constant relaxation time approximation. The details of the calculations are shown in the Supplemental Material (SM) [49]. At large $|\alpha|$ and low energies, both systems show a similar behavior: the conductivity of isolated Weyl nodes is $\sigma_{j j} \propto \mu^{2}$ [50], with $j=x, y, z$ and $\mu$ the chemical potential measured relative to the Weyl node energy. At $\alpha=0$, however, the two systems are significantly different. In the three-node process we have a $\mathcal{C}=-1$ node, with linear dispersion in $k_{x}$ and $k_{y}$ and cubic dispersion in the $k_{z}$ direction. In the two-node process we have a double Weyl point with $\mathcal{C}=-2$ and with quadratic dispersion in $k_{x}$ and $k_{z}$, but linear dispersion in $k_{y}$. This difference in the dispersion at low energies results in different conductivities as a function of chemical potential. At $\alpha=0$ the three-node process is characterized by

$$
\sigma_{x x} \propto \mu^{4 / 3}, \quad \sigma_{y y} \propto \mu^{4 / 3}, \quad \sigma_{z z} \propto \mu^{8 / 3},
$$

whereas the two-node process shows

$$
\sigma_{x x} \propto \mu^{2}, \quad \sigma_{y y} \propto \mu, \quad \sigma_{z z} \propto \mu^{2} .
$$

Note that in the latter case we only focused on the double Weyl node forming at $k_{z}=0$. The third Weyl node will contribute additively to the conductivity (see SM [49]).

These differences are prominent when looking at the conductivities in the plane of the three nodes $\left(\sigma_{x x}\right.$ and $\left.\sigma_{z z}\right)$. In Fig. 2 we plot the ratio $\sigma_{x x} / \sigma_{z z}$ as a function of $\alpha$ for different chemical potential values. Panel (a) shows the three-node process, in which the ratio is enhanced close to $\mu=0$ and $\alpha=0$, which is due to the different dispersions in the two directions. On the contrary, panel (b) shows that $\sigma_{x x} / \sigma_{z z}=1$ at $\alpha=0$ for the two-node process, because of the identical dispersion relations in the two directions.

Weyl nodes in strained $\mathrm{MoTe}_{2}$. Many materials reported to host Weyl cones have $C_{2} T$ symmetry, and in several cases Weyl nodes are placed inside the $C_{2} T$-invariant plane, including $\{\mathrm{W}, \mathrm{Mo}\} \mathrm{Te}_{2}[38,51], \mathrm{Ta}\{\mathrm{Ir}, \mathrm{Rh}\} \mathrm{Te}_{4}$ [52], and $\mathrm{ZrTe}$ [53]. Under a suitable external perturbation, these compounds are natural candidates for the three-node process described above. As an example, we show based on density-functional calculations that uniaxial strain in $\mathrm{MoTe}_{2}$ controls a process of the type sketched in Fig. 1(b). We perform fully relativistic calculations as implemented in the FPLO code [54]. We use as equilibrium lattice parameters those reported in Ref. [38] 


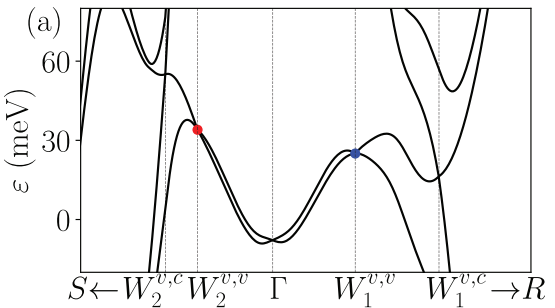

(b)
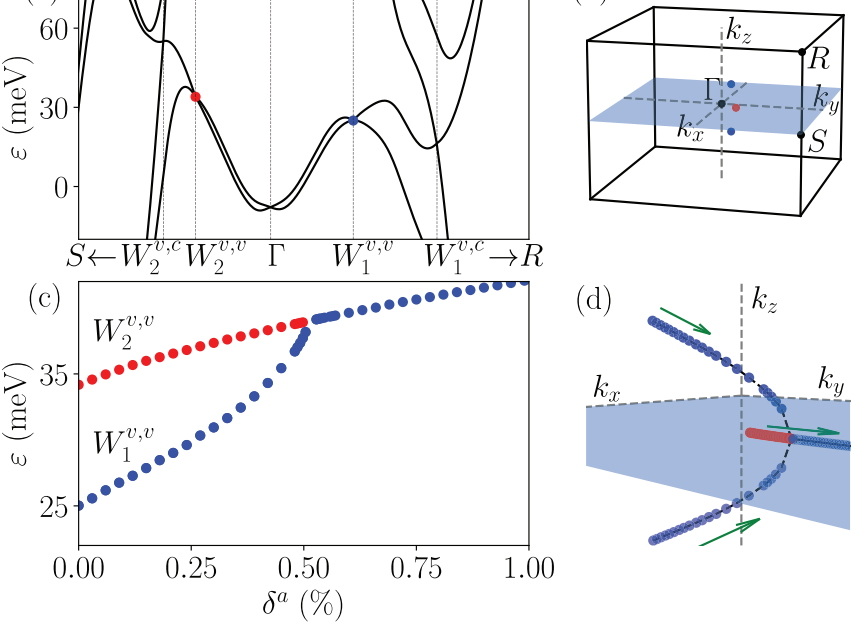

FIG. 3. (a) Band structure of $\mathrm{MoTe}_{2} \cdot W_{1}^{v, c}\left(W_{2}^{v, c}\right)$ is a Weyl node outside (inside) the $k_{z}=0$ plane, and connects valence and conducting bands. Analogously, $W_{1}^{v, v}$ and $W_{2}^{v, v}$ connect the two upper valence bands. Blue (red) corresponds to negative (positive) chirality. (b) Brillouin zone including the Weyl nodes $W_{1}^{v, v}$ and $W_{2}^{v, v}$ having positive $k_{x}, k_{y}$ coordinates. The $k_{z}=0$ plane is indicated in blue. (c),(d) Energy and momentum trajectories of $W_{1}^{v, v}$ and $W_{2}^{v, v}$ as a function of tensile strain. Green arrows indicate the trajectories upon increasing the tensile strain.

at $100 \mathrm{~K}(a=3.468 \AA, b=6.31 \AA$, and $c=13.861 \AA)$. We focus on uniaxial strain such that the lattice parameter $a$ is enlarged. For a fixed deformation $\delta^{a}$ (measured in percent of the original value of $a$ ), we determine the deformations of $b$ and $c$ according to the Poisson ratios $\gamma_{a b}=0.19$ and $\gamma_{a c}=0.96$ reported in Ref. [55]. Previous works have shown that treating the Mo- $4 d$ shell with the GGA $+\mathrm{U}$ method improves the description of photoemission experiments provided by GGA $[31,51,56,57]$. Thus we use here the former method with $J=0$ and $U=2.6 \mathrm{eV}$. Further details, including the robustness of our results to all these choices, are presented in the SM [49].

Figure 3(a) shows the band structure of $\mathrm{MoTe}_{2}$ without strain $\left(\delta^{a}=0\right)$ along a path that includes Weyl nodes connecting the higher valence and lower conducting bands $\left(W_{1}^{v, c}\right.$ and $W_{2}^{v, c}$ ) and nodes between the two upper valence bands $\left(W_{1}^{v, v}\right.$ and $\left.W_{2}^{v, v}\right)$. The results of interest in this work originate in the latter two which are found $25 \mathrm{meV}\left(W_{1}^{v, v}\right)$ and $34 \mathrm{meV}$ $\left(W_{2}^{v, v}\right)$ above the Fermi level. Note that both are type II, or overtilted nodes, unlike the band touching points of the toy models presented above. $W_{1}^{v, v}$ has seven other partners in the BZ, which together form a set of eight nodes at generic momentum coordinates, which are related to each other by the reflection symmetries $M_{x}$ and $M_{y}$, the twofold rotation around the $c$ axis, $C_{2}$, and time reversal $T$. On the other hand, there are only four nodes of the type $W_{2}^{v, v}$, all of which are positioned inside the $C_{2} T$-invariant plane [Fig. 3(b)]. As $\delta^{a}$ increases, the energy difference between $W_{1}^{v, v}$ and $W_{2}^{v, v}$ decreases and vanishes at the critical point $\delta_{c}^{a} \approx 0.5 \%$ [Fig. 3(c)].

Within our numerical precision, at $\delta_{c}^{a}$ the Weyl nodes $W_{1}^{v, v}$ are annihilated, while the nodes $W_{2}^{v, v}$ change their chirality. There are a total of four such processes throughout the entire $\mathrm{BZ}$, one at each quadrant of the $\left(k_{x}, k_{y}\right)$ plane, due to the presence of reflection symmetries. In a given quadrant, before the Weyl merging, one finds a pair of nodes at finite $k_{z}$ and Chern number $\mathcal{C}=-1$, and a single node having $k_{z}=0$ and $\mathcal{C}=1$. After the merging, only a single Weyl node having $\mathcal{C}=-1$ and $k_{z}=0$ exists [Fig. 3(d)]. Thus $\mathrm{MoTe}_{2}$ realizes a three-node process of the type discussed previously.

Note that none of the nodes are on the twofold rotation axis, so the triple-Weyl merging is not forced to occur, i.e., it is not protected by symmetry. Instead, our results suggest that the three-node process is favored energetically, since it involves a smaller change in the local gap between the bands forming the Weyl cones (see SM [49]).

Conclusion. We have shown that the topological charge associated to Weyl cones can change sign. This chirality flip involves three nodes and may occur generically in Weyl semimetal phases. We discussed the two generic mechanisms by which this can happen: a three-node process, where a triplet of Weyl cones merge at the same point in the BZ, and a two-node process, which occurs by means of successive, pairwise mergings of nodes. Additional symmetries, in our case time-reversal and twofold rotation, increase the likelihood of chirality flips by constraining the positions and relative charges of Weyl nodes. In some cases, symmetries may even enforce the occurrence of the three-node process.

Our results indicate that the chirality flip occurs in one of the most well-studied Weyl materials, $\mathrm{MoTe}_{2}$, where moderate uniaxial strain leads to a simultaneous merging of three nodes close to the Fermi level. Depending on the doping level of different $\mathrm{MoTe}_{2}$ samples, this indicates that chirality flips are within reach of photoemission and transport experiments. It could be interesting to extend our study of electronic transport to models relevant to $\mathrm{MoTe}_{2}$, in particular to magnetotransport properties sensitive to the chiral anomaly.

More generally, our work sets the stage for further investigating such chirality converting processes in a wide range of materials and scenarios. As we have mentioned, there is an abundance of Weyl materials obeying $C_{2} T$ symmetry, many of which host Weyl cones pinned to the $C_{2} T$-invariant plane. Furthermore, similar processes could be also observed in topological metamaterials that host Weyl nodes such as interacting spin systems [58,59] or multiterminal Josephson junctions $[60,61]$.

An interesting direction for future work could be to examine the behavior of chiral topological metals, which may obey time-reversal and rotation symmetries, but in which inversion symmetry is strongly broken. In these systems, Kramers' theorem guarantees the presence of Weyl points at the timereversal invariant momenta (TRIM) of the BZ. When TRIM points lie on a rotation axis, we have shown that chirality flips must occur via a three-node process.

Acknowledgments. We thank U. Nitzsche for technical assistance and J. K. Asbóth for useful discussions. This work was supported by the Deutsche Forschungsgemeinschaft (DFG, German Research Foundation) under Germany's Excellence Strategy through the Würzburg-Dresden Cluster of Excellence on Complexity and Topology in Quantum Matter-ct.qmat (EXC 2147, project-id 390858490). R.-J. S. acknowledges funding from the Marie Skłodowska-Curie 
programme under EC Grant No. 842901 and the Winton programme as well as Trinity College at the University of
Cambridge. J.I.F. acknowledges the support from the Alexander von Humboldt Foundation.
[1] H. B. Nielsen and M. Ninomiya, Absence of neutrinos on a lattice. (I). Proof by homotopy theory, Nucl. Phys. B 185, 20 (1981).

[2] S. Murakami, Phase transition between the quantum spin Hall and insulator phases in 3D: Emergence of a topological gapless phase, New J. Phys. 9, 356 (2007).

[3] X. Wan, A. M. Turner, A. Vishwanath, and S. Y. Savrasov, Topological semimetal and Fermi-arc surface states in the electronic structure of pyrochlore iridates, Phys. Rev. B 83, 205101 (2011).

[4] A. A. Burkov and L. Balents, Weyl Semimetal in a Topological Insulator Multilayer, Phys. Rev. Lett. 107, 127205 (2011).

[5] A. A. Burkov, M. D. Hook, and L. Balents, Topological nodal semimetals, Phys. Rev. B 84, 235126 (2011).

[6] C. K. Chiu, J. C. Y. Teo, A. P. Schnyder, and S. Ryu, Classification of topological quantum matter with symmetries, Rev. Mod. Phys. 88, 035005 (2016).

[7] B. Yan and C. Felser, Topological materials: Weyl semimetals, Annu. Rev. Condens. Matter Phys. 8, 337 (2017).

[8] N. P. Armitage, E. J. Mele, and A. Vishwanath, Weyl and Dirac semimetals in three-dimensional solids, Rev. Mod. Phys. 90, 015001 (2018).

[9] B. A. Bernevig, H. Weng, Z. Fang, and X. Dai, Recent progress in the study of topological semimetals, J. Phys. Soc. Jpn. 87, 041001 (2018).

[10] H. Weyl, Elektron und gravitation. I, Z. Phys. 56, 330 (1929).

[11] A. A. Soluyanov, D. Gresch, Z. Wang, Q. Wu, M. Troyer, X. Dai, and B. A. Bernevig, Type-II Weyl semimetals, Nature (London) 527, 495 (2015).

[12] S. M. Young, S. Zaheer, J. C. Y. Teo, C. L. Kane, E. J. Mele, and A. M. Rappe, Dirac Semimetal in Three Dimensions, Phys. Rev. Lett. 108, 140405 (2012).

[13] Z. Wang, Y. Sun, X. Q. Chen, C. Franchini, G. Xu, H. Weng, $\mathrm{X}$. Dai, and Z. Fang, Dirac semimetal and topological phase transitions in $A_{3} \mathrm{Bi}(A=\mathrm{Na}, \mathrm{K}, \mathrm{Rb})$, Phys. Rev. B 85, 195320 (2012).

[14] B. Bradlyn, J. Cano, Z. Wang, M. G. Vergniory, C. Felser, R. J. Cava, and B. A. Bernevig, Beyond Dirac and Weyl fermions: Unconventional quasiparticles in conventional crystals, Science 353, aaf5037 (2016).

[15] L. Lepori, I. C. Fulga, A. Trombettoni, and M. Burrello, PTinvariant Weyl semimetals in gauge-symmetric systems, Phys. Rev. B 94, 085107 (2016).

[16] L. Lepori, I. C. Fulga, A. Trombettoni, and M. Burrello, Double Weyl points and Fermi arcs of topological semimetals in nonAbelian gauge potentials, Phys. Rev. A 94, 053633 (2016).

[17] I. C. Fulga and A. Stern, Triple point fermions in a minimal symmorphic model, Phys. Rev. B 95, 241116(R) (2017).

[18] C. Fang, H. Weng, X. Dai, and Z. Fang, Topological nodal line semimetals, Chin. Phys. B 25, 117106 (2016).

[19] E. J. Sie, C. M. Nyby, C. D. Pemmaraju, S. J. Park, X. Shen, J. Yang, M. C. Hoffmann, B. K. Ofori-Okai, R. Li, A. H. Reid, S. Weathersby, E. Mannebach, N. Finney, D. Rhodes, D. Chenet, A. Antony, L. Balicas, J. Hone, T. P. Devereaux, T. F. Heinz et al., An ultrafast symmetry switch in a Weyl semimetal, Nature (London) 565, 61 (2019).

[20] J. Liu and D. Vanderbilt, Weyl semimetals from noncentrosymmetric topological insulators, Phys. Rev. B 90, 155316 (2014).

[21] R.-J. Slager, V. Juričić, V. Lahtinen, and J. Zaanen, Selforganized pseudo-graphene on grain boundaries in topological band insulators, Phys. Rev. B 93, 245406 (2016).

[22] J. I. Facio, D. Efremov, K. Koepernik, J.-S. You, I. Sodemann, and J. van den Brink, Strongly Enhanced Berry Dipole at Topological Phase Transitions in BiTeI, Phys. Rev. Lett. 121, 246403 (2018).

[23] C. L. Zhang, S. Y. Xu, C. M. Wang, Z. Lin, Z. Z. Du, C. Guo, C. C. Lee, H. Lu, Y. Feng, S. M. Huang, G. Chang, C. H. Hsu, H. Liu, H. Lin, L. Li, C. Zhang, J. Zhang, X. C. Xie, T. Neupert, M. Z. Hasan et al., Magnetic-tunnelling-induced Weyl node annihilation in TaP, Nat. Phys. 13, 979 (2017).

[24] J. Cano, B. Bradlyn, Z. Wang, M. Hirschberger, N. P. Ong, and B. A. Bernevig, Chiral anomaly factory: Creating Weyl fermions with a magnetic field, Phys. Rev. B 95, 161306(R) (2017).

[25] M. P. Ghimire, J. I. Facio, J.-S. You, L. Ye, J. G. Checkelsky, S. Fang, E. Kaxiras, M. Richter, and J. van den Brink, Creating Weyl nodes and controlling their energy by magnetization rotation, Phys. Rev. Research 1, 032044(R) (2019).

[26] R. Ray, B. Sadhukhan, M. Richter, J. I. Facio, and J. van den Brink, Tunable chirality of noncentrosymmetric magnetic Weyl semimetals, arXiv:2006.10602.

[27] R.-J. Slager, V. Juričić, and B. Roy, Dissolution of topological Fermi arcs in a dirty Weyl semimetal, Phys. Rev. B 96, 201401(R) (2017).

[28] B. Roy, R.-J. Slager, and V. Juričić, Global Phase Diagram of a Dirty Weyl Liquid and Emergent Superuniversality, Phys. Rev. X 8, 031076 (2018).

[29] J. H. Pixley, D. A. Huse, and S. Das Sarma, Rare-RegionInduced Avoided Quantum Criticality in Disordered ThreeDimensional Dirac and Weyl Semimetals, Phys. Rev. X 6, 021042 (2016).

[30] J. H. Pixley and J. H. Wilson, Rare regions and avoided quantum criticality in disordered Weyl semimetals and superconductors, Ann. Phys. (NY) 168455 (2021), doi: 10.1016/j.aop.2021.168455.

[31] S. Singh, J. Kim, K. M. Rabe, and D. Vanderbilt, Engineering Weyl Phases and Nonlinear Hall Effects in $\mathrm{T}_{d}-\mathrm{MoTe}_{2}$, Phys. Rev. Lett. 125, 046402 (2020).

[32] P. H. Fu, H. J. Duan, R. Q. Wang, and H. Chen, Phase transitions in three-dimensional Dirac semimetal induced by off-resonant circularly polarized light, Phys. Lett. A 381, 3499 (2017).

[33] P. H. Fu, J. Wang, J. F. Liu, and R. Q. Wang, Josephson signatures of Weyl node creation and annihilation in irradiated Dirac semimetals, Phys. Rev. B 100, 115414 (2019).

[34] C. Fang, M. J. Gilbert, X. Dai, and B. A. Bernevig, Multi-Weyl Topological Semimetals Stabilized by Point Group Symmetry, Phys. Rev. Lett. 108, 266802 (2012). 
[35] I. C. Fulga, L. Fallani, and M. Burrello, Geometrically protected triple-point crossings in an optical lattice, Phys. Rev. B 97, 121402(R) (2018).

[36] S. Thirupathaiah, Y. S. Kushnirenk, K. Koepernik, B. R. Piening, B. Buechner, S. Aswartham, J. van den Brink, S. Borisenko, and I. C. Fulga, Sixfold fermion near the Fermi level in cubic PtBi2, SciPost Phys. 10, 004 (2021).

[37] Q. Xu, Y. Zhang, K. Koepernik, W. Shi, J. van den Brink, C. Felser, and Y. Sun, Comprehensive scan for nonmagnetic Weyl semimetals with nonlinear optical response, Npj Comput. Mater. 6, 1 (2020).

[38] A. Tamai, Q. S. Wu, I. Cucchi, F. Y. Bruno, S. Riccò, T. K. Kim, M. Hoesch, C. Barreteau, E. Giannini, C. Besnard, A. A. Soluyanov, and F. Baumberger, Fermi Arcs and Their Topological Character in the Candidate Type-II Weyl Semimetal $\mathrm{MoTe}_{2}$, Phys. Rev. X 6, 031021 (2016).

[39] We calculate the Chern number using the occupied bands with the Berry curvature defined as $\mathbf{B}_{n, k}=\nabla_{k} \times \mathbf{A}_{n, k}$, where $\mathbf{A}_{n, k}=$ $-i\left\langle n, k\left|\nabla_{k}\right| n, k\right\rangle$. This definition is chosen to be consistent with the FPLO code.

[40] A. Bouhon, A. M. Black-Schaffer, and R.-J. Slager, Wilson loop approach to fragile topology of split elementary band representations and topological crystalline insulators with time-reversal symmetry, Phys. Rev. B 100, 195135 (2019).

[41] A. Bouhon, G. F. Lange, and R.-J. Slager, Topological correspondence between magnetic space group representations and subdimensions, Phys. Rev. B 103, 245127 (2021).

[42] F. N. Ünal, A. Bouhon, and R.-J. Slager, Topological Euler Class as a Dynamical Observable in Optical Lattices, Phys. Rev. Lett. 125, 053601 (2020).

[43] J. Ahn, S. Park, and B.-J. Yang, Failure of Nielsen-Ninomiya Theorem and Fragile Topology in Two-Dimensional Systems with Space-Time Inversion Symmetry: Application to Twisted Bilayer Graphene at Magic Angle, Phys. Rev. X 9, 021013 (2019).

[44] B. Jiang, A. Bouhon, Z.-K. Lin, X. Zhou, B. Hou, F. Li, R.-J. Slager, and J.-H. Jiang, Experimental observation of nonAbelian topological acoustic semimetals and their phase transitions, Nat. Phys. (2021), doi: 10.1038/s41567-021-01340-x.

[45] A. Bouhon, T. Bzdušek, and R.-J. Slager, Geometric approach to fragile topology beyond symmetry indicators, Phys. Rev. B 102, 115135 (2020).

[46] Q. Wu, A. A. Soluyanov, and T. Bzdušek, Non-Abelian band topology in noninteracting metals, Science 365, 1273 (2019).

[47] B. Peng, A. Bouhon, B. Monserrat, and R.-J. Slager, Non-Abelian braiding of phonons in layered silicates, arXiv:2105.08733.

[48] A. Bouhon, Q. Wu, R.-J. Slager, H. Weng, O. V. Yazyev, and T. Bzdušek, Non-Abelian reciprocal braiding of Weyl points and its manifestation in ZrTe, Nat. Phys. 16, 1137 (2020).

[49] See Supplemental Material at http://link.aps.org/supplemental/ 10.1103/PhysRevResearch.3.L042017 for (i) details of the con- ductivity calculation, (ii) details of the ab initio results, and (iii) animations of the Weyl-node dynamics as obtained from the model Hamiltonians and from the $a b$ initio calculations. Input files used in this study as well as relevant data are available in the repository at [62].

[50] C. J. Tabert, J. P. Carbotte, and E. J. Nicol, Optical and transport properties in three-dimensional Dirac and Weyl semimetals, Phys. Rev. B 93, 085426 (2016).

[51] N. Xu, Z. W. Wang, A. Magrez, P. Bugnon, H. Berger, C. E. Matt, V. N. Strocov, N. C. Plumb, M. Radovic, E. Pomjakushina, K. Conder, J. H. Dil, J. Mesot, R. Yu, H. Ding, and M. Shi, Evidence of a Coulomb-Interaction-Induced Lifshitz Transition and Robust Hybrid Weyl Semimetal in $T_{d}-\mathrm{MoTe}_{2}$, Phys. Rev. Lett. 121, 136401 (2018).

[52] K. Koepernik, D. Kasinathan, D. V. Efremov, S. Khim, S. Borisenko, B. Büchner, and J. van den Brink, TaIrTe ${ }_{4}$ : A ternary type-II Weyl semimetal, Phys. Rev. B 93, 201101(R) (2016).

[53] H. Weng, C. Fang, Z. Fang, and X. Dai, Coexistence of Weyl fermion and massless triply degenerate nodal points, Phys. Rev. B 94, 165201 (2016).

[54] K. Koepernik and H. Eschrig, Full-potential nonorthogonal local-orbital minimum-basis band-structure scheme, Phys. Rev. B 59, 1743 (1999).

[55] J. Yang, J. Colen, J. Liu, M. C. Nguyen, G.-w. Chern, and D. Louca, Elastic and electronic tuning of magnetoresistance in $\mathrm{MoTe}_{2}$, Sci. Adv. 3, eaao4949 (2017).

[56] F.-T. Huang, S. J. Lim, S. Singh, J. Kim, L. Zhang, J.-W. Kim, M.-W. Chu, K. M. Rabe, D. Vanderbilt, and S.-W. Cheong, Polar and phase domain walls with conducting interfacial states in a Weyl semimetal $\mathrm{MoTe}_{2}$, Nat. Commun. 10, 1 (2019).

[57] N. Aryal and E. Manousakis, Importance of electron correlations in understanding photoelectron spectroscopy and Weyl character of MoTe2, Phys. Rev. B 99, 035123 (2019).

[58] Z. Scherübl, A. Pályi, G. Frank, I. E. Lukács, G. Fülöp, B. Fülöp, J. Nygård, K. Watanabe, T. Taniguchi, G. Zaránd, and S. Csonka, Observation of spinorbit coupling induced Weyl points in a two-electron double quantum dot, Commun. Phys. 2, 108 (2019).

[59] G. Frank, Z. Scherübl, S. Csonka, G. Zaránd, and A. Pályi, Magnetic degeneracy points in interacting two-spin systems: Geometrical patterns, topological charge distributions, and their stability, Phys. Rev. B 101, 245409 (2020).

[60] B. van Heck, S. Mi, and A. R. Akhmerov, Single fermion manipulation via superconducting phase differences in multiterminal Josephson junctions, Phys. Rev. B 90, 155450 (2014).

[61] R. P. Riwar, M. Houzet, J. S. Meyer, and Y. V. Nazarov, Multi-terminal Josephson junctions as topological matter, Nat. Commun. 7, 11167 (2016).

[62] V. Könye, A. Bouhon, I. C. Fulga, R.-J. Slager, J. van den Brink, and J. I. Facio, Chirality flip of Weyl nodes and its manifestation in strained $\mathrm{MoTe}_{2}$, Zenodo (2021). 